\title{
FAIR BANDWIDTH ALLOCATION FOR DIFFERENTIATED SERVICES
}

\author{
Anindya Basu and Zheng Wang \\ Bell Laboratories \\ \{basu,zhwang\}@research.bell-lab.com
}

\begin{abstract}
In this paper, we present a comparative study of three proposals for differentiated services - the RIO scheme, the two-bit scheme and the UserShare Differentiation (USD) scheme. We simulate the three schemes under a number of different scenarios including mismatching of bottleneck bandwidth and profiles, short duration traffic, and non-responsive sources. Our findings show that although the simpler schemes for Differentiated Services reduce the complexity in the core, they tend to perform less well when there is a mismatch between the allocated profiles and bottleneck bandwidth, and also when the traffic sources are bursty, and non-responsive.
\end{abstract}

\section{INTRODUCTION}

Over the last ten years, considerable effort has been made to provide Quality-of-Services (QoS) guarantees in the Internet. Much of the work has focused on the end-to-end per-session reservation approach where an application makes an end-to-end reservation before starting a session [16]. While it makes sense to set up a reservation for a long-lasting session, this approach is not suitable for short duration, and transaction-oriented applications such as browsing the World Wide Web where the overheads and latency involved in setting up a reservation for each session could become disproportionately high. Furthermore, there are also concerns regarding the scalability of providing guarantees at the per-session level in the core of the Internet.

The above issues have led to a number of new proposals for providing differentiated services in the Internet [1]. In contrast to the per-session reservation approach, the differentiated services approach allows service providers to offer different levels of services to a few (typically, a small number such as two or three) classes of aggregated traffic flows. Examples of this approach include the RIO scheme by Clark et al [2] and the two-bit scheme by Nichols et al [11]. They advocate pushing traffic policing and admission

The original version of this chapter was revised: The copyright line was incorrect. This has been corrected. The Erratum to this chapter is available at DOI: 10.1007/978-0-387-35580-1_16 
control to the edge of the network and leave the core of the network as simple as possible.

With such an approach, Incoming traffic from a user is monitored at the edge of the network against the profile that the user has registered (and paid for) with the service provider. The packets that are out of profile are either shaped or tagged and the core routers process such tagged (or untagged) packets according to predefined policies. Such an approach has the advantage of simplicity since core routers do not need to store state for each traffic flow; instead, they can process packets using different policies for each traffic class which is determined by a small number of bits in the packet header.

A different set of proposals that is exemplified by the User-Share Differentiation (USD) scheme proposed by Wang [15] advocate putting modestly sophisticated control mechanisms in core routers to provide proportional fair sharing (PFS) of resources and per-prefix traffic isolation. The advantage of such schemes is that they do not require a priori estimation of resources that a particular user or an application expects to use. At any point in the network, the USD scheme can ensure that the bandwidth allocated to a flow is commensurate with some predefined metric, such as the price paid by the originator of the flow to the ISP. For example, it is possible to guarantee that user A who pays twice as much as user B, gets twice as much bandwidth as user B anywhere in a network. The major disadvantage of such schemes lie in the increased complexity of the routers in the network core and the concern that such complexity may not be amenable to scaling.

In this paper, we present a comparative study of the three proposals for differentiated services, namely the RIO scheme, the 2-bit scheme and the User-Share Differentiation (USD) scheme. We implement each of these schemes on a simulated network using the REAL network simulator [9] and study their performances under different network configurations. We find that the USD scheme provides a better match of performance to user expectations and is more robust with respect to short-lived flows such as web traffic, but this comes at the added cost of implementing PFS at the network core.

The rest of the paper is organized as follows: Section 2 describes the three schemes in detail. Section 3 evaluates the performance of the three schemes under different scenarios, followed by Section 4 that summarizes the results of the evaluation. We conclude the paper in Section 5 with a discussion of some of the implications of our findings.

\section{THREE DIFFSERV SCHEMES}

In this section, we describe in more detail the three differentiated services schemes, namely, the RIO scheme, the two-bit scheme and the USD scheme. 
The RIO scheme (RIO stands for Random Early Detection with In/Out bit) uses some form of packet tagging to indicate the drop priority of the packet to the core network routers. Each user (or traffic flow, depending upon the granularity) is assigned a service profile by the ISP based on the expected bandwidth utilization by the user. At the edge of the network domain managed by the ISP (i.e., at the ingress points), user traffic is monitored by a profile meter to ensure that it stays within the profile. Any packets that are out of profile are marked as "out" while those that conform to the user profile are marked as "in". In the network core, the "in" and "out" packets are treated with different drop priorities using RED (Random Early Detection).

Since the "in" packets start being dropped only when the queue size crosses a higher threshold than in the case of "out" packets, they get dropped with a lower probability than "out" packets. This ensures that in-profile traffic has less chance of getting dropped than out-of-profile traffic, and therefore gets predictable levels of service so long as it stays within profile (even when congestion occurs). The RIO scheme also makes it possible to use statistical multiplexing to utilize any excess bandwidth that may be available since it does not prevent out-of-profile packets from entering the network.

The two-bit differentiated services architecture combines the RIO scheme with Jacobson's premium service [8] to come up with three different classes of service, namely premium, assured and best-effort. The premium service is based on explicit resource reservations and a simple priority queuing model where the premium traffic is transmitted prior to other traffic. Such a service is useful for time-critical applications that require minimum delay as well as applications that require explicit bandwidth and delay guarantees.

The premium traffic is strictly policed at the edge of the network: packets that are out of the service profile are dropped or delayed until they are within the service profile. The assured service class corresponds to the "in" traffic in the RIO scheme and has a lower drop priority than the ordinary best-effort service. When congestion occurs, the chances are high that the assured packets would receive predictable levels of service if they conform to their service profile, which is typically based on average bandwidth utilization. This service is appropriate for users who are willing to pay somewhat more in order to get better (or predictable) performance in times of congestion.

The USD scheme takes an approach that is different from that of the RIO and two-bit schemes. First, instead of taking a minimalist approach with a small number of distinguishable classes, the USD scheme allows traffic isolation on a per-customer basis. This allows more sophisticated service 
contracts to be supported. Second, in contrast to the RIO and two-bit schemes where the traffic policing happens at the edge of the network, the USD scheme manages bandwidth allocation directly on the bottlenecks where the congestion takes place. Finally, while both RIO and two-bit schemes primarily deal with outgoing traffic traveling from customers' networks to their ISP, the USD scheme works with traffic of both outgoing and incoming directions.

The USD scheme introduces two terms for bandwidth allocation, user and share. The user refers to the customer to which the bandwidth is allocated. In the USD scheme, a user can be a network, a group of networks identified by a prefix, or an individual end user. Each user is assigned a number called share, based on some predefined metrics, such as the amount that a user has paid for the service. The USD scheme operates with the proportional fair sharing (PFS) principle, where at any point in a network, the bandwidth allocated to the traffic from or to a user is in proportion to the user's share. In other words, the exact amount of bandwidth allocated to flow from or to a user is determined by the total bandwidth available at the bottleneck and the relative weights (or shares) of active users competing for the bottleneck resources.

The USD scheme can be implemented with a classifier capable of prefix lookup (to identify flows belonging to individual customers) and a scheduler that supports PFS. Prefix lookup has been extensively studied for route lookups and several novel algorithms have been proposed for this purpose [5]. Hardware-based route lookup implementations that can support over 1 millions entries [10] exist and the same techniques can be used in the USD for identifying which customer a packet belongs to. A large number of scheduling algorithms can support PFS. Among them, weighted fair queuing (WFQ) [12] can support PFS with the most stringent fairness guarantees. Many variations of WFQ, such as Deficit Round Robin (DRR) and Self-Clocked Fair Queuing (SCFQ), have lower implementation complexity and yet provide reasonable fairness guarantees [7,13]. For example, the PacketStar Router from Bell Labs implements a variant of WFQ with 64,000 queues at $622 \mathrm{Mb} / \mathrm{s}$ speed [10].

\section{SIMULATING DIFFERENTIATED SERVICES}

In this section, we present and discuss the simulation results for the three differentiated services proposals described in the previous section. While the ideal method of doing a comparative study would be to deploy each of the three schemes on the internet, we relied on simulations instead since deploying new mechanisms on a real network without a thorough understanding of the ramifications would be inappropriate. These simulations are intended to provide us with a detailed description of how the network 
behaves when such schemes are deployed. Through such a process, we hope to gain a deeper understanding of the strengths and weaknesses of these schemes before they are actually deployed on a real network.

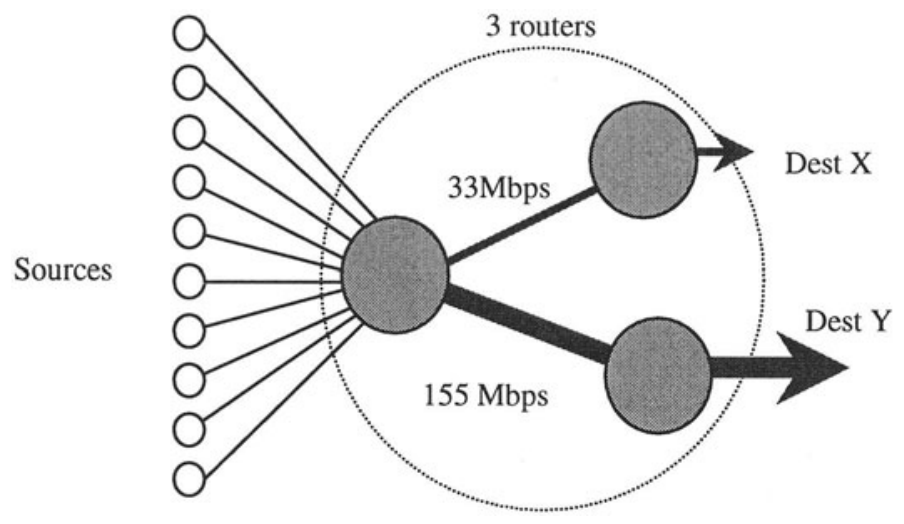

Figure 1: Simulated Network

The simulations were done with the REAL network simulator [9]. For purposes of the simulation, we used a network with the configuration shown in Figure 1. In the simulation, we have 10 sources (1 through 10 counting downwards) that communicate with one of two different destinations, $\mathrm{X}$ and $\mathrm{Y}$. The sources are connected to destination $\mathrm{X}$ through a bottleneck link of capacity $33 \mathrm{Mbps}$ and to destination $\mathrm{Y}$ through a bottleneck link of capacity $155 \mathrm{Mbps}$. Each of the links that connects a source to the first router in the diagram has a capacity of $30 \mathrm{Mb} / \mathrm{s}$. The one-way propagation delays (the same to both $\mathrm{X}$ and $\mathrm{Y}$ ) for the sources (counting from top downwards) were set at $20,20,40,40,50,50,70,70,100$, and 100 milliseconds respectively. The sources are all TCP-Reno sources (unless specified otherwise) that use a packet size of 500 bytes and each of them sends 500,000 packets to the destination.

For the RIO implementation, the routers use RED with the values of 400 packets, 800 packets, and 0.02 for min_in, max_in, and $P_{\max \_i n}$, and 100 packets, 200 packets and 0.5 for min_out, max_out, and $P_{\text {max_out }}[2]$ where $\min \_$in and max_in represent the upper and lower bounds for the average queue size for in profile packets when RED kicks in and $P_{\text {max } i n}$ is the maximum drop probability for an in profile packet when the average queue size is in the [min_in, max_in] range. The min_out, max_out and $P_{\text {max_out }}$ are the corresponding parameters for the out of profile packets. Note that the total queue sizes are used when calculating the drop probability for out of profile packets, as suggested in [2]. 
For the USD scheme, we used the weighted fair queuing (WFQ) discipline on the router to provide PFS. While it can be argued that WFQ was not the most appropriate scheme to use for this purpose which advocates use of WFQ variants with less implementation complexity and less strict guarantees), we feel that the results provide an important baseline for purposes of comparison with the other schemes.

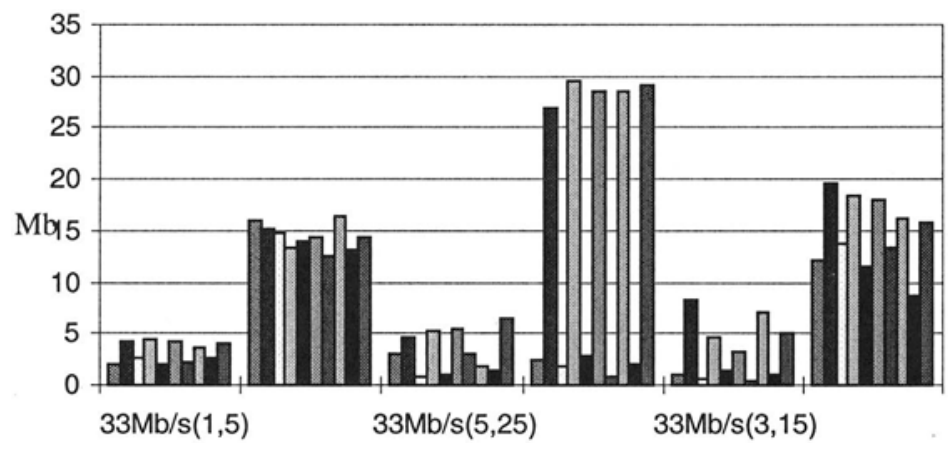

Figure 1: Bandwidth share with two different bottleneck bandwidths and 3 different expected profiles using the RIO scheme



Figure 2: Bandwidth share with two different bottleneck bandwidths and 3 different expected profiles using the two-bit scheme.

\section{Effect of Expected Bandwidth Profiles}

We first evaluated the effect of expected bandwidth profiles and bottleneck bandwidths on actual bandwidth allocation. For this purpose, we ran 
three sets of simulations for each of the two bottleneck links $(33 \mathrm{Mb} / \mathrm{s}$ to destination $\mathrm{X}$ and $155 \mathrm{Mb} / \mathrm{s}$ to destination $\mathrm{Y}$ ). In each of the simulations, the odd numbered sources $(1,3,5,7,9)$ had the lower expected bandwidth and the even numbered sources $(2,4,6,8,10)$ had the higher expected bandwidth. In the first set of simulations, we set the expected lower and higher bandwidths to $1 \mathrm{Mb} / \mathrm{s}$ and $5 \mathrm{Mb} / \mathrm{s}$, respectively, such that the aggregate (i.e., sum of) expected bandwidth of all the flows matched the $33 \mathrm{Mb} / \mathrm{s}$ bottleneck bandwidth. In the second set, the expected bandwidths were chosen to be 5 and $25 \mathrm{Mb} / \mathrm{s}$, respectively to match the $155 \mathrm{Mb} / \mathrm{s}$ bottleneck and in the third set, they were set to 3 and $15 \mathrm{Mb} / \mathrm{s}$, respectively, which is the average of expected bandwidths in the first two sets.

The performance of the RIO scheme is shown in Figure 1. The figure consists of six clusters with 10 bars in each cluster. Each cluster has a label of the form $x \mathrm{Mb} / \mathrm{s}(y, z)$, which means that the particular cluster represents the bandwidth allocation for the 10 sources when the bottleneck bandwidth is $x \mathrm{Mb} / \mathrm{s}$ and the higher and lower expected bandwidths are $y$ and $z \mathrm{Mb} / \mathrm{s}$, respectively. The results indicate that if the aggregate expected bandwidth of all the flows passing through the bottleneck closely matches the bottleneck

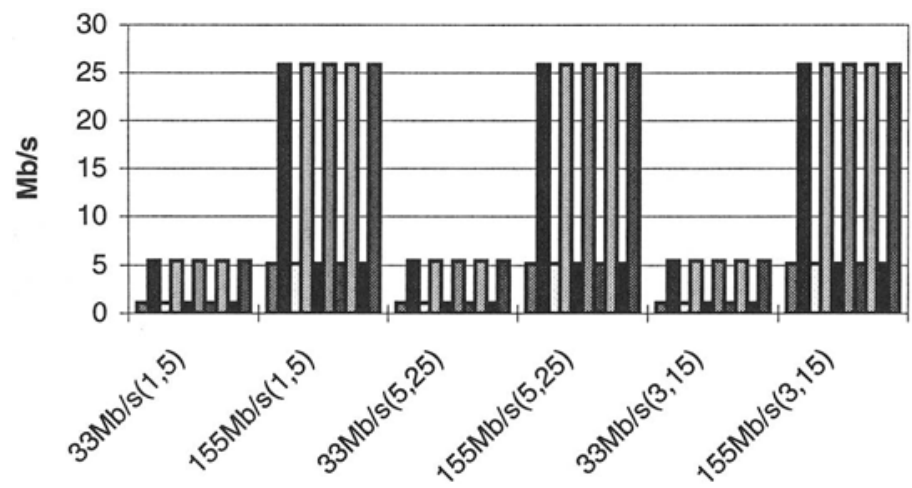

Figure 3: Bandwidth share with two different bottleneck bandwidths and 3 different expected profiles using the USD scheme

bandwidth, the RIO scheme is able to allocate bandwidth in accordance with the expected bandwidth profiles. This is clear from the graphs for the $33 \mathrm{Mb} / \mathrm{s}$ $(1,5)$ and $155 \mathrm{Mb} / \mathrm{s}(5,25)$ cases. In the first case, the sources with a lower expected bandwidth are allocated a slightly higher bandwidth than their expected profiles at the expense of sources with a higher expected bandwidth and the situation is the reverse in the $155 \mathrm{Mb} / \mathrm{s}(5,25)$ case. We expect that some tuning of the RED parameters is required to obtain a bandwidth allocation that is more commensurate with the expected bandwidth profiles. If the 
bottleneck bandwidth is in excess of the aggregated expected bandwidth, the excess bandwidth is more or less evenly allocated among all the sources. This is evident from the figures for the $155 \mathrm{Mb} / \mathrm{s}(3,15)$ and the $155 \mathrm{Mb} / \mathrm{s}(1,5)$ cases. In the $155 \mathrm{Mb} / \mathrm{s}(3,15)$ case, the sources with the higher expected bandwidth do get more bandwidth than the others, but the relative difference is smaller than those in the $33 \mathrm{Mb} / \mathrm{s}(1,5)$ and the $155 \mathrm{Mb} / \mathrm{s}(5,25)$ cases where the bottleneck bandwidth matches the aggregate expected bandwidth. In the $155 \mathrm{Mb} / \mathrm{s}(1,5)$ case (where the difference between the bottleneck bandwidth and the aggregated expected bandwidth is higher), the bandwidth allocation is fairly even among all the sources. This is because the excess bandwidth is only allocated to the out of profile packets, and the RED scheme for out of profile packets ensures that this excess bandwidth is allocated fairly. Finally, if the aggregated expected bandwidth is less than the bottleneck bandwidth, the RIO scheme is able (for the most part) to allocate the existing bandwidth in a manner that is commensurate with existing user expectations. There is one exception in the $33 \mathrm{Mb} / \mathrm{s}(5,25)$ case, where the bandwidth allocated to source 8 is less than that allocated to sources 1 and 7 although the latter have lower expected bandwidths. In summary, the RIO scheme performs well if the bottleneck bandwidth matches or is less than the aggregate expected bandwidth of all the flows passing through the bottleneck, but is unable to allocate bandwidth in proportion to user expectations if there is excess bandwidth at the bottleneck.

To evaluate the two-bit scheme, we set the higher bandwidth traffic as the premium traffic. For example, in the $33 \mathrm{Mb} / \mathrm{s}(1,5)$ case, the $5 \mathrm{Mb} / \mathrm{s}$ sources were all considered premium sources with a peak sending rate of $5 \mathrm{Mb} / \mathrm{s}$. Network bandwidth for premium traffic was reserved at connection set up time. The low bandwidth sources were subjected to the RIO scheme where all the in profile packets had their A-bit set while the out of profile packets did not. The RIO parameters in this case were the same as in the previous experiment. Figure 2 shows the bandwidth allocation for the two-bit scheme. We see that the bandwidth allocation matches the expected bandwidth in the $33 \mathrm{Mb} / \mathrm{s}(1,5)$ case and the $155 \mathrm{Mb} / \mathrm{s}(5,25)$ case. This implies that the two-bit scheme performs well when the bottleneck bandwidth matches the aggregate expected bandwidth. However, we see that in the $155 \mathrm{Mbit} / \mathrm{s}$ $(1,5)$ and the $155 \mathrm{Mb} / \mathrm{s}(3,15)$ cases, the bandwidth share allocated to the sources with the lower expected bandwidth is higher. This is because of how premium traffic is handled in the network: any out of profile premium packet is dropped by the profile meter. Consequently, all the excess bandwidth in these cases goes to the sources with lower expected bandwidth. In this respect, the bandwidth allocation done by the RIO scheme is more in keeping with user expectations. In the $33 \mathrm{Mb} / \mathrm{s}(5,25)$ and $33 \mathrm{Mb} / \mathrm{s}(3,15)$ cases, some of the high expected bandwidth sources do not get any bandwidth at all. This 
is because their connection set up calls get rejected due to unavailable bandwidth. Thus, for the two-bit scheme with premium traffic, it is important that the aggregated expected bandwidth through the bottleneck match the bottleneck bandwidth, else, some premium traffic may get denied bandwidth because the network resources have been over committed.

Finally, we simulated the USD scheme using weighted fair queuing in the network core. The bandwidth allocation is shown in Figure 3. In this case, we see that the relative bandwidth allocated to all the sources remain the same in all cases. In other words, the sources with high expected bandwidth (which presumably have paid more for such a privilege) get a higher relative share of the bandwidth than the others, independent of the bottleneck bandwidth or the absolute values of the expected bandwidth profiles.

\section{Short Duration Traffic}

A significant component of Internet traffic today is web traffic [14] that is characterized by short connection lifetimes. In order to study the effect of short connection lifetimes on the three different schemes, we ran simulations with the same settings as before except that the sources now sent only 200 packets to the destination. This corresponds to a web transfer of $100 \mathrm{Kbytes}$, a moderately sized web page with embedded graphics. Figure 4 shows the performance for the $33 \mathrm{Mb} / \mathrm{s}(1,5)$ (or matching bottleneck bandwidth) scenario and Figure 5 shows the performance for the $155 \mathrm{Mb} / \mathrm{s}(1,5)$ (or excess bottleneck bandwidth) scenario. The numbers for the $33 \mathrm{Mb} / \mathrm{s}(1,5)$ scenario show

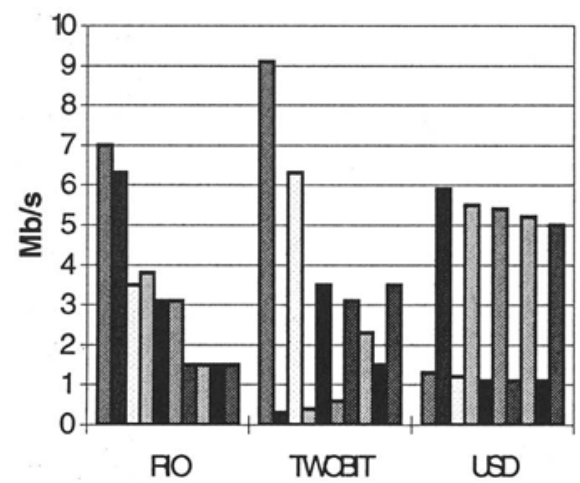

Figure 4: Short-duration traffic with $33 \mathrm{Mb} / \mathrm{s}(1,5)$

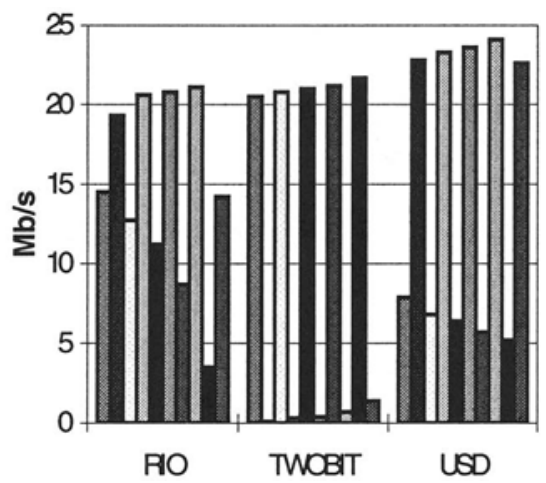

Figure 5: Short-duration traffic with $155 \mathrm{Mb} / \mathrm{s}(1,5)$

that on such short time scales, the bandwidth allocated by the RIO scheme is not commensurate with the expected bandwidth profiles. This is because the RED technique used in the routers for estimating average queue lengths (that determines when to drop a packet) and the Time Sliding Window technique 
used by a profile meter to estimate the sending rate for a source (that determines whether a packet is in or out of profile) depend on long term averages. In the shorter term, the Time Sliding Window technique tends to overestimate the sending rate and mark more packets as out of profile than is necessary. We have performed some experiments to test how sensitive the calculated sending rates are to the length of the time window (the results are not shown here for lack of space), and found that the closer the time windows are to the round trip time, the more accurate the estimates are. This implies that it may be difficult to estimate sending rates accurately in the absence of information about round-trip times, which is the case if the profile meter is decoupled from the sender. In the $155 \mathrm{Mb} / \mathrm{s}(1,5)$ case, the bandwidth allocation is more reasonable in the sense that the higher bandwidth sources receive greater bandwidth than the lower bandwidth ones, except for source 10 that gets slightly lower bandwidth than source 1 . However, the bandwidth allocations are not in proportion to the respective expected average bandwidths. We have seen earlier (section 0) that the bandwidth at the bottleneck that is in excess of the aggregate expected bandwidth is more or less evenly allocated among all the sources - in this case, where all the traffic flows are of much shorter duration, RED is unable to enforce fair sharing of the excess bandwidth. The higher bandwidth sources, in the short term, are able to grab a higher share of the excess bandwidth since their congestion windows open up faster (due to lower drop rates at the routers that implement RIO) than those of the lower bandwidth sources.

The numbers for the two-bit scheme are interesting. The even sources (i.e., those with expected bandwidths of $5 \mathrm{Mb} / \mathrm{s}$ ), for the most part, show a lower bandwidth allocation compared to the odd sources (i.e. those with expected bandwidths of $1 \mathrm{Mb} / \mathrm{s}$ ) in both the scenarios. This anomalous behavior is caused by the way premium traffic is managed under the two-bit scheme: the monitor at the edge of the network generates premium tokens at a fixed rate, based on the peak bandwidth reserved by the call set up mechanism. Since TCP-Reno starts by doubling the size of the congestion window every round trip time, the traffic generated is bursty and the sending rate can exceed the reserved bandwidth in the short term. As a result, the premium traffic, on reaching the monitor, faces an absence of premium tokens and gets dropped. This happens in the initial stages of the opening up of the congestion window (before ssthresh is reached) and fast recovery does not occur because there are not enough lost packets. Thus, TCP times out multiple times and causes the congestion window to open up very slowly. In the long run, this effect is absent because over longer time scales, enough premium tokens are generated to absorb such bursts. Thus, in the short term, the sources with the lower expected bandwidth are able to make use of the excess bandwidth that is freed up by a lack of premium traffic and therefore 
show higher bandwidth utilization. In the case of the USD scheme, the short term bandwidth allocations match the long term case: the figures in each of the two scenarios show that the bandwidth shares for the sources are in proportion to their expected bandwidth profiles. Thus, for short-lived flows such as web traffic, where end-to-end mechanisms are harder to implement because they need at least one round trip time to react, USD is an attractive option for providing differentiated services.

\section{Non-Responsive Sources}

We used two different kinds of non-responsive sources, CBR (Constant Bit Rate) sources that send at a fixed rate and malicious sources that flood the network by sending as fast as they can. None of these sources back off in the face of congestion. We first investigated the effects of CBR sources on each of the three schemes. The simulations were repeated using bottleneck links of capacity $33 \mathrm{Mb} / \mathrm{s}$ and $155 \mathrm{Mb} / \mathrm{s}$. In the simulations, sources 1 and 2 were CBR sources transmitting at $5 \mathrm{Mb} / \mathrm{s}$ and the other sources were TCPReno sources where the odd numbered sources had an expected bandwidth of $1 \mathrm{Mb} / \mathrm{s}$ and the even numbered sources had an expected bandwidth of $5 \mathrm{Mb} / \mathrm{s}$. All CBR traffic was marked as out of profile.

Figure 6 shows the bandwidth allocation in each of the three schemes when the bottleneck bandwidth is $33 \mathrm{Mb} / \mathrm{s}$ and Figure 7 shows the same when the bottleneck bandwidth is $155 \mathrm{Mb} / \mathrm{s}$. In both figures, the first two bars in

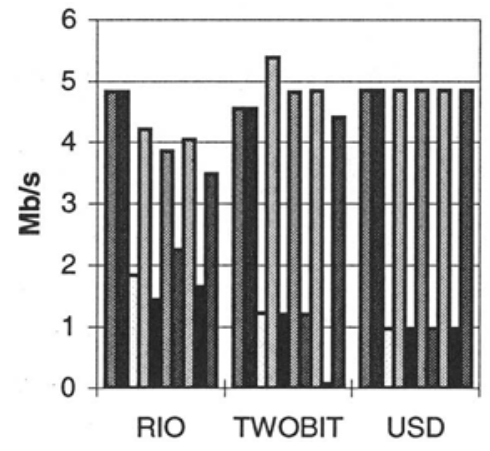

Figure 6: Effect of two nonresponsive (CBR) sources with $33 \mathrm{Mb} / \mathrm{s}(1,5)$



Figure 7: Effect of two non-responsive (CBR) sources with $155 \mathrm{Mb} / \mathrm{s}(1,5)$

each cluster are for the CBR sources and the rest are for the TCP-Reno sources. The numbers for the $33 \mathrm{Mb} / \mathrm{s}(1,5)$ case indicate that in the case of RIO, the CBR sources are able to get close to their expected bandwidth (i.e., about $5 \mathrm{Mb} / \mathrm{s}$ ) despite all of their traffic being marked out of profile (i.e. lower priority). However, the TCP-Reno sources perform reasonably well, 
the sources with higher expected bandwidth show a bandwidth share of about $4 \mathrm{Mb} / \mathrm{s}$ which is close to what the expected bandwidth profile is. The sources with a lower expected bandwidth show bandwidth shares that are higher than their expected bandwidth profiles, closer to about $2 \mathrm{Mb} / \mathrm{s}$ rather than $1 \mathrm{Mb} / \mathrm{s}$. As in section 0 , we expect that this can be remedied by an appropriate adjustment of the RIO parameters. In the $155 \mathrm{Mb} / \mathrm{s}(1,5)$ case, the CBR sources get about $5 \mathrm{Mb} / \mathrm{s}$ as in the previous case. All the other sources (that are all TCP-Reno sources) receive bandwidths that are in excess of their expected average bandwidths, and it is clear that the excess bandwidth at the bottleneck is allocated fairly evenly among the TCP sources by the RED mechanism, as was the case in Section 0.

For the two-bit scheme, the CBR sources get close to their expected bandwidth (about $4.5 \mathrm{Mb} / \mathrm{s}$ ) in both the scenarios. The sources with the higher expected high bandwidth, whose packets are all marked as premium traffic, also get close to $5 \mathrm{Mb} / \mathrm{s}$ bandwidth (about $4.8 \mathrm{Mb} / \mathrm{s}$ from the graphs). In the $33 \mathrm{Mb} / \mathrm{s}(1,5)$ case, 3 of the 4 sources that have a low expected bandwidth profile of $1 \mathrm{Mb} / \mathrm{s}$, get a slightly higher bandwidth allocation than their expected profiles. All of this comes at the expense of the fourth low bandwidth source, which gets almost close to zero bandwidth. Thus, it appears that in

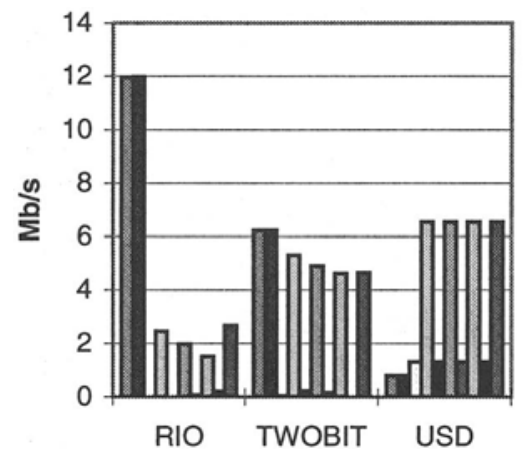

Figure 8: Two malicious sources with $33 \mathrm{Mb} / \mathrm{s}(1,5)$

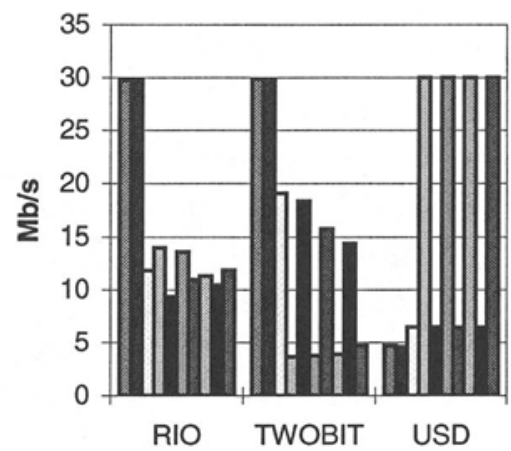

Figure 9: Two malicious sources with $155 \mathrm{Mb} / \mathrm{s}(1,5)$

the two-bit scheme, if there are non-cooperative, bandwidth hungry sources (even if all their packets are marked out of profile), other well-behaved, low priority traffic may get starved. The situation is slightly different when there is excess bandwidth at the bottleneck, i.e., the $155 \mathrm{Mb} / \mathrm{s}(1,5)$ case. In this scenario, the premium bandwidth sources do not get any more than their expected bandwidths because premium traffic that is out of profile gets dropped. Consequently, the excess bandwidth at the bottleneck is shared evenly by the low bandwidth TCP-Reno sources, that now show a higher 
bandwidth allocation than the higher bandwidth sources. Finally, the numbers for the USD scheme show that the bandwidth allocations are commensurate with the expected bandwidth profiles, which implies that the USD scheme is effective in the presence of non co-operative CBR sources.

To investigate the effect of malicious sources, we again used bottleneck bandwidths of $33 \mathrm{Mb} / \mathrm{s}$ and $155 \mathrm{Mb} / \mathrm{s}$. Sources 1 and 2 were set to be malicious sources that declare an expected bandwidth of $1 \mathrm{Mb} / \mathrm{s}$ and then try to flood the network by sending as fast as they can. The other sources are TCPReno sources where the odd numbered sources have an expected bandwidth of $1 \mathrm{Mb} / \mathrm{s}$ and the even numbered sources have an expected bandwidth of $5 \mathrm{Mb} / \mathrm{s}$. Note that these scenarios are different from the CBR cases described earlier, because in the CBR cases, the sources did not try to flood the network in addition to not responding to congestion. The purpose of simulating malicious sources here is to see if they have a negative effect on low priority traffic by filling up the queues allocated for such traffic.

The results for the $33 \mathrm{Mb} / \mathrm{s}(1,5)$ scenario are shown in Figure 8 . In the RIO case, the low bandwidth sources get very little bandwidth - sources 3 and 5 get nothing and sources 7 and 9 get about $0.1 \mathrm{Mb} / \mathrm{s}$. The high bandwidth sources do not do well either, none of the sources are able to get more than $2.5 \mathrm{Mb} / \mathrm{s}$. The reason for this is that the packets for the malicious sources fill up the queues on the bottleneck routers very quickly and causes packets from other sources to be dropped more often. Consequently, the TCP windows do not open up to the extent required to get the expected average bandwidth. For the two-bit scheme, the low bandwidth sources get very little bandwidth (as in the RIO case) for the same reason and the bulk of the bandwidth goes to the malicious sources. However, in this case, the malicious sources grab about $6 \mathrm{Mb} / \mathrm{s}$ each as opposed to $12 \mathrm{Mb} / \mathrm{s}$ each in the RIO case. The reason is that the packets from the high bandwidth sources in the two-bit scheme are enqueued in a separate, higher priority, premium traffic queue. As a result, the high bandwidth sources are able to get close to their expected bandwidths of $5 \mathrm{Mb} / \mathrm{s}$ while the low bandwidth sources get very little - sources 3, 5 and 7 get less than $0.2 \mathrm{Mb} / \mathrm{s}$ and source 9 gets nothing. Thus, in both RIO and two-bit schemes, a malicious source can easily deprive low priority traffic of their fair share, but the high priority traffic tends to do better in the two-bit scheme because such traffic is enqueued in a separate, high priority queue. In the case of the USD scheme, the malicious sources are penalized and actually receive slightly less than their average expected bandwidth $(0.79 \mathrm{Mb} / \mathrm{s}$ in place of $1 \mathrm{Mb} / \mathrm{s})$ while the other sources get slightly more than their expected bandwidth - the low bandwidth sources get $1.31 \mathrm{Mb} / \mathrm{s}$ instead of $1 \mathrm{Mb} / \mathrm{s}$ and the high bandwidth sources get $6.54 \mathrm{Mb} / \mathrm{s}$ instead of $5 \mathrm{Mb} / \mathrm{s}$. 
The results for the $155 \mathrm{Mb} / \mathrm{s}(1,5)$ scenario are shown in Figure 9. In this scenario, the malicious sources for the RIO and the two-bit schemes are able to grab almost the full bandwidth of their outgoing links, which is set to $30 \mathrm{Mb} / \mathrm{s}$ in the simulation. In the RIO case, the TCP-Reno sources are able to get more than their expected bandwidth profiles, but not in proportion to their expected bandwidths. Again, the reason is that the excess bandwidth (that is consumed only by out of profile packets) is more or less evenly distributed by the RED scheme among all the sources. In the two-bit case, the premium sources are able to get slightly less than their expected bandwidths (in the range $3.5-4 \mathrm{Mb} / \mathrm{s}$ instead of $5 \mathrm{Mb} / \mathrm{s}$ ). This is somewhat surprising since, given that the bottleneck bandwidth in this case is $155 \mathrm{Mb} / \mathrm{s}$, and the fact that premium traffic gets higher priority than best effort traffic, one would have expected that the premium traffic would get the full $5 \mathrm{Mb} / \mathrm{s}$ bandwidth. We conjecture that this is because the malicious sources constantly fill up the lower priority queues and therefore the routers spend more time in processing the lower priority queues than the higher priority ones. Consequently, the congestion windows for the higher bandwidth premium traffic does not open up fast enough to utilize the requisite expected bandwidth. The excess bottleneck bandwidth is more or less evenly allocated only among the low bandwidth sources because any premium traffic that is out of profile is dropped. This causes the low bandwidth sources to get a higher bandwidth allocation than the high bandwidth sources. Lastly, the USD scheme again penalizes the malicious sources and the allocation of bandwidth among the well behaved TCP-Reno sources is in proportion to their expected average bandwidths the figures show that the low bandwidth sources get about $6 \mathrm{Mb} / \mathrm{s}$ while the high bandwidth sources get about $30 \mathrm{Mb} / \mathrm{s}$.

\section{SUMMARY}

In this paper, we have evaluated the bandwidth allocation performance of the RIO, the two-bit and the USD schemes. We have shown that matching the network bottleneck bandwidth to the aggregated expected bandwidth profiles over all the traffic flows through the bottleneck is an important issue. While the RIO and the two-bit schemes perform well when there is no mismatch, such is not the case if the bottleneck bandwidth available is in excess of the aggregated expected bandwidth through the bottleneck. The RIO and two-bit schemes need to be modified in some way to react more quickly to short-lived flows. In the case of non-responsive sources, RIO performs better than the two-bit scheme when the non-responsive sources are CBR, however, malicious sources that flood the network are able to starve low priority traffic. The USD scheme is able to perform well in all scenarios. 


\section{REFERENCES}

[1] D. Black, S. Blake, M. Carlson, E. Davies, Z. Wang, and W. Weiss. An Architecture for Differentiated Services, RFC 2475, May 1998.

[2] D. Clark and W. Fang. Explicit Allocation of Best Effort Packet Delivery Service, Work in progress, 1998.

[3] D. Clark, S. Shenker, and L. Zhang. Supporting Real-Time Applications in an Integrated Services Packet Network: Architecture and Mechanisms, In Proceedings of ACM SIGCOMM'92, August 1992.

[4] D. Clark, J. Wroclawski. An Approach to Service Allocation in the Internet, Work in progress, July 1997.

[5] M. Degermark, A. Brodnok, S. Carlsson, and S. Pink. Small Forwarding Tables for Fast Routing Lookups, In Proceedings of ACM SIGCOMM'97, Cannes, France, September 1997.

[6] K. Fall and S. Floyd. Promoting the Use of End-to-End Congestion Control in the Internet, Work in progress, February 1998.

[7] S. Golestani. A self-clocked fair queuing scheme for broadband applications, In Proceedings of IEEE INFOCOM'94, pages 636-646, April 1994.

[8] V. Jacobson. Differentiated Services Architecture, Presented at the IETF meeting at the Munich IETF, August 1997.

[9] S. Keshav. REAL 5.0 Manuals, http://www.cs.cornell.edu/skeshav/real/man.html, August 1997.

[10] V. P. Kumar, T. V. Lakshman, and D. Stiliadis. Beyond Best Effort: Router Architectures for the Differentiated Services of Tomorrow's Internet, IEEE Communications Magazine, May 1998.

[11] K. Nichols, V. Jacobson, and L. Zhang. A Two-bit Differentiated Services Architecture for the Internet, Work in progress, November 1997.

[12] A. K. Parekh and R. G. Gallager, "A Generalized Processor Sharing Approach to Flow Control - the Single Node Case, In Proceedings of IEEE INFOCOM'92, May 1992.

[13] M. Shreedhar and G. Varghese. Efficient Fair Queuing Using Deficit Round Robin, In Proceedings of ACM SIGCOMM'95, Cambridge, Massachusetts, August 1995.

[14] K. Thompson, G. Miller, and R. Wilder. Wide-Area Internet Traffic Patterns and Characteristics, IEEE Network, November 1997.

[15] Z. Wang. USD: Scalable Bandwidth Allocation for the Internet, In Proceedings of HPN'98, September 1998.

[16] L. Zheng, S. Deering, S. Estrin, S. Shenker, and D. Zappala. A New Resource Reservation Protocol, IEEE Network, September 1993. 